\title{
Interacting Dark Matter as an Alternative to Dark Energy
}

\author{
Spyros Basilakos* and Manolis Plionis ${ }^{\dagger}$ \\ *Academy of Athens, Research Center for Astronomy and Applied Mathematics, Soranou Efesiou 4, 11527, \\ Athens, Greece \\ ${ }^{\dagger}$ Institute of Astronomy \& Astrophysics, National Observatory of Athens, Palaia Penteli 152 36, Athens, Greece \\ Instituto Nacional de Astrofísica, Óptica y Electronica, Puebla, Mexico
}

\begin{abstract}
We investigate the global dynamics of the universe within the framework of the Interacting Dark Matter (IDM) scenario. Considering that the dark matter obeys the collisional Boltzmann equation, we can obtain analytical solutions of the global density evolution, which can accommodate an accelerated expansion, equivalent to either the quintessence or the standard $\Lambda$ models. This is possible if there is a disequilibrium between the DM particle creation and annihilation processes with the former process dominating, which creates an effective source term with negative pressure. Comparing the predicted Hubble expansion of one of the IDM models (the simplest) with observational data, we find that the effective annihilation term is quite small, as suggested by various experiments.
\end{abstract}

Keywords: Cosmology; dark matter; dark energy

PACS: 98.80.-k, 95.35.+d,95.36.+x

\section{INTRODUCTION}

The detailed analysis of the available high quality cosmological observations ([1, 2, 3, 4] and references therein) have converged during the last decade towards a cosmic expansion history that involves a spatial flat geometry and a recent accelerating expansion of the universe. This expansion has been attributed to an energy component (the so called dark energy) with negative pressure which dominates the universe at late times and causes the observed accelerating expansion. The nature of the dark energy is still a mystery and it is one of the most fundamental current problems in physics and cosmology. Indeed, due to the absence of a physically well-established fundamental theory, there have been many theoretical speculations regarding the nature of the above exotic dark energy (DE) among which a cosmological constant, scalar or vector fields (see [5, 6, 7, 8, 9, 10, 11] and references therein).

Most of the recent papers in this kind of studies are based on the assumption that the DE evolves independently of the dark matter (DM). The unknown nature of both DM and DE implies that we can not preclude future surprises regarding the interactions in the dark sector. This is very important because interactions between the DM and quintessence could provide possible solutions to the cosmological coincidence problem. Recently, several papers have been published in this area [12, 13] proposing that the DE and DM could be coupled, assuming also that there is only one type of non-interacting DM.

However, there are other possibilities. (a) It is plausible that the dark matter is self-interacting (IDM) [14], a possibility that has been proposed to solve discrepancies between theoretical predictions and astrophysical observations, among which the gamma-ray and microwave emission from the center of our galaxy (eg. [15, 16], [17, 18] and references therein). It has also been shown that some dark matter interactions could provide an accelerated expansion phase of the Universe [19, 20, 21]. (b) The DM could potentially contain more than one particle species, for example a mixture of cold and warm or hot dark matter [22, 23], with or without inter-component interactions.

In this work we are not concerned with the viability of the different such possibilities, nor with the properties of interacting DM models. The aim of this work is to investigate only whether there are repercussions of DM selfinteractions for the global dynamics of the universe and specifically whether such models can yield an accelerated phase of the cosmic expansion, without the need of the dark energy. We note that we do not "design" the fluid interactions to produce the desired accelerated cosmic evolution, as in some previous works (eg., [20]), but investigate the circumstances under which the analytical solution space of the collisional Boltzmann equation, in the expanding universe, allows for a late accelerated phase of the universe. 


\section{COLLISIONAL BOLTZMANN EQUATION IN THE EXPANDING UNIVERSE}

As we described in the introduction the DM is usually considered to contain only one type of particles that are stable and neutral. In this work, we would like to investigate the cosmological potential of a different scenario, in which the dominant "cosmic" fluid does not contain dark energy, is not perfect, and at the same time is not in equilibrium. In this context, initially the total energy density is $\rho=\rho_{\mathrm{IDM}}+\rho_{r}$. We consider that the self-interacting dark matter does not interact significantly with the background radiation, and thus in the matter-dominated epoch, radiation is irrelevant to the global dynamics (because of the well-known dependence: $\rho_{r} \propto a^{-4}$ ). Therefore, taking the latter considerations into account and assuming that there are no residual radiation products of the DM interactions (otherwise see [24]), we conclude that in the matter-dominated era the total cosmic dark-matter density reduces to that of the IDM density ( $\rho \simeq \rho_{\text {IDM }}$ ), which obeys the collisional Boltzmann equation [25]:

$$
\frac{\mathrm{d} \rho}{\mathrm{d} t}+3 H(t) \rho+\kappa \rho^{2}-\Psi=0,
$$

where $H(t) \equiv \dot{\alpha} / \alpha$ is the Hubble function, $\Psi$ is the rate of creation of DM particle pairs, and $\kappa(\geq 0)$ is given by:

$$
\kappa=\frac{\langle\sigma u\rangle}{M_{x}},
$$

where $\sigma$ is the cross-section for annihilation, $u$ is the mean particle velocity, and $M_{x}$ is the mass of the DM particle. It is obvious that if we impose in the current analysis an effective pressure term such as

$$
P=\left(\kappa \rho^{2}-\Psi\right) / 3 H,
$$

then the collisional Boltzmann equation reduces to the usual fluid equation: $\dot{\rho}+3 H(\rho+P)=0$. Now if the DM is collisionless or the collisional annihilation and pair creation processes are in equilibrium (ie., $P=0$ ), the corresponding solution of the above differential equation is $\rho \propto \alpha^{-3}$, as it should (where $\alpha$ is the scale factor of the universe). In contrast, for the case of a non-perfect fluid (ie., having a disequilibrium between the annihilation and particle pair creation processes) it becomes clear that we can either have a positive or a negative effective pressure term.

The possibility of a negative such effective pressure term, ie., the case for which the DM particle creation term is larger than the annihilation term $\left(\kappa \rho^{2}-\Psi<0\right)$, is of particular interest for its reprecursions on the global dynamics of the Universe (see appendix A). In the framework of a Boltzmann formalism, a negative pressure could in general be the outcome of dark matter self-interactions, as suggested in [19, 20], if an "anti-frictional" force is self-consistently exerted on the particles of the cosmic fluid. This possible alternative to dark energy has the caveat of its unknown exact microphysics, which is also however the case for all dark energy models. Note, that in the literature, there is a wealth of papers discussing the DM particle creation processes in the context of thermodynamics (for example, eg. [26]):

We now proceed in an attempt to analytically solve eq. (1). To this end we change variables from $t$ to $\alpha$ and thus eq.(1) can be written:

$$
\frac{\mathrm{d} \rho}{\mathrm{d} \alpha}=f(\alpha) \rho^{2}+g(\alpha) \rho+R(\alpha)
$$

where

$$
f(\alpha)=-\frac{\kappa}{\alpha H(\alpha)} \quad g(\alpha)=-\frac{3}{\alpha} \quad R(\alpha)=\frac{\Psi(\alpha)}{\alpha H(\alpha)} .
$$

In general it is not an easy task to solve analytically eq. (4), which is a Riccati equation, due to the fact that it is a non-linear differential equation. However, eq. (4) could be fully solvable if (and only if) a particular solution is known. Indeed, we find that for some special cases regarding the functional form of the interactive term, such as $\Psi=\Psi(\alpha, H)$, we can derive analytical solutions (see appendix B). We have phenomenologically identified two functional forms for which we can solve the previous differential equation analytically, only one of which is of interest since it provides $\propto a^{-3}$ dependence of the scale factor. This is:

$$
\Psi(\alpha)=\alpha H(\alpha) R(\alpha)=C_{1}(m+3) \alpha^{m} H(\alpha)+\kappa C_{1}^{2} \alpha^{2 m} .
$$

Although, the above functional form was not motivated by some physical theory, but rather phenomenologically because it provides analytical solutions to the Boltzmann equation, its exact form can be justified a posteriori within the framework of IDM (see Appendix C). 
The general solution of equation (4) using eq.(6) for the total energy density is:

$$
\rho(\alpha)=C_{1} \alpha^{m}+\frac{\alpha^{-3} F(\alpha)}{\left[C_{2}-\int_{1}^{\alpha} x^{-3} f(x) F(x) d x\right]},
$$

where the kernel function $F(\alpha)$ has the form

$$
F(\alpha)=\exp \left[-2 \kappa C_{1} \int_{1}^{\alpha} \frac{x^{m-1}}{H(x)} d x\right]
$$

We note that $\kappa C_{1}$ has units of $\mathrm{Gyr}^{-1}$, while $m, C_{1}$, and $C_{2}$ are the corresponding constants of the problem. Obviously, Eq.(7) can be rewritten as

$$
\rho(\alpha)=\rho_{c}(\alpha)+\rho^{\prime}(\alpha),
$$

where $\rho_{c}=C_{1} \alpha^{m}$ is the density corresponding to the residual matter creation that results from a possible disequilibrium between the particle creation and annihilation processes, while $\rho^{\prime}$ can be viewed as the energy density of the selfinteracting dark matter particles that are dominated by the annihilation processes. We note that close to the present epoch as well as at late enough times $(\alpha \gg 1)$, the $\rho^{\prime}$ evolves in a similar way to the usual dark matter (see also [27]). Finally, if both $\kappa$ and $\Psi$ tend to zero, the above cosmological model reduces to the usual Einstein-deSitter model $\left(\rho \propto a^{-3}\right)$.

We note that, since $\rho^{\prime}>0$, the constant $C_{2}$ obeys the restriction

$$
C_{2}>G(\alpha)=\int_{1}^{\alpha} x^{-3} f(x) F(x) d x \geq 0 .
$$

Evaluating now Eq.(7) at the present time $(\alpha=1, F(\alpha)=1)$, we obtain the present-time total cosmic density, which is: $\rho_{0}=C_{1}+1 / C_{2}$, with $C_{1} \geq 0$ and $C_{2}>0$.

\section{Conditions to have an inflection point}

Using the Hubble function: $H^{2}(\alpha)=(\dot{\alpha} / \alpha)^{2}=8 \pi G \rho(\alpha) / 3$, we derive the second derivate of the scale factor:

$$
\frac{\ddot{\alpha}}{\alpha}=-\frac{4 \pi G}{3}\left(-2 \rho-\frac{\dot{\rho}}{H}\right) \text {. }
$$

Inserting Eqs.(1) and (3) into Eq.(11), we obtain

$$
\frac{\ddot{\alpha}}{\alpha}=-\frac{4 \pi G}{3}\left(\rho+\frac{\kappa \rho^{2}-\Psi}{H}\right)=-\frac{4 \pi G}{3}(\rho+3 P) .
$$

In order to have an inflection point ( $\ddot{\alpha}=0)$, the expression $\rho+3 P=0$ should contain a real root in the interval: $\alpha \in(0,1)$. Thus, with the aid of Eq.(7), (3) and (6), we derive the following condition:

$$
\frac{\alpha^{-3} F\left(H+2 \kappa C_{1} \alpha^{m}\right)}{C_{2}-G}+\frac{\kappa \alpha^{-6} F^{2}}{\left(C_{2}-G\right)^{2}}-(m+2) C_{1} \alpha^{m} H=0
$$

from which we obtain that $m>-2$ (where $C_{1}>0, \kappa \geq 0$, and $C_{2}-G>0$ ). Evidently, if we parametrize the constant $m$ according to $m=-3\left(1+w_{\text {IDM }}\right)$, we obtain the condition $w_{\text {IDM }}<-1 / 3$, which implies that the current cosmological model can be viewed as a viable quintessence dark-energy look-alike, as far as the global dynamics is concerned. We remind the reader that the same restriction holds for the conventional dark energy model in which $P_{Q}=w \rho_{Q}$ $(w=$ const $<-1 / 3)$. 

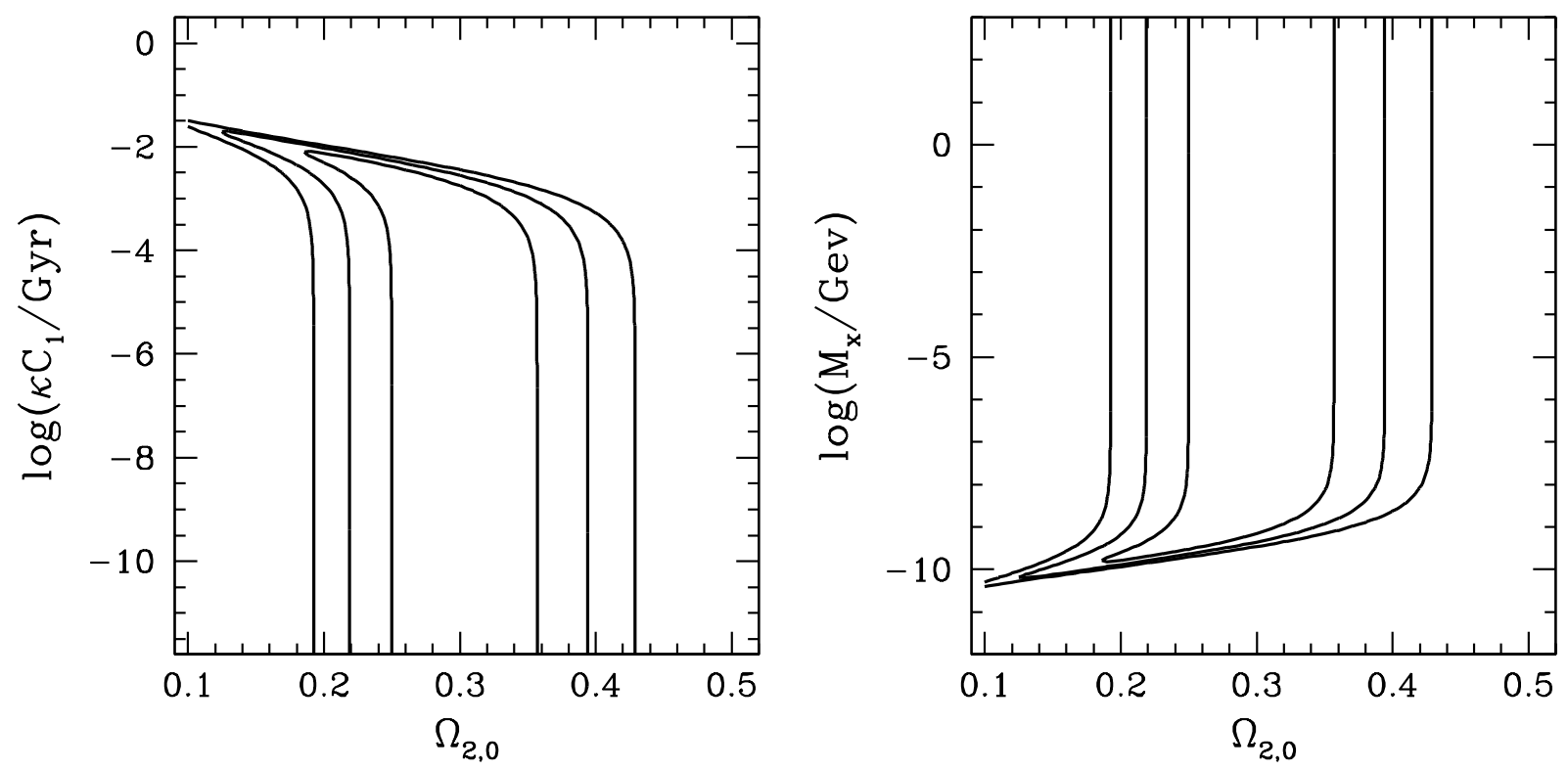

FIGURE 1. Left panel: The $\Omega_{2,0}-\kappa C_{1}$ solution space provided by fitting our model to the early-type galaxy Hubble relation of Simon et al (2005). Right panel: The corresponding $\Omega_{2,0}-M_{x}$ solution space.

\section{MIMICKING THE $\Lambda$ COSMOLOGY}

We will show that for $m=0$ the global dynamics, provided by our model (see eq.7), is equivalent to that of the traditional $\Lambda$ cosmology. Indeed, we use $\mathrm{d} t=\mathrm{d} \alpha /(\alpha H)$ and the basic kernel (Eq. 8) becomes

$$
F(\alpha)=\exp \left[-2 \kappa C_{1} \int_{1}^{\alpha} \frac{1}{x H(x)} d x\right]=e^{-2 \kappa C_{1}\left(t-t_{0}\right)},
$$

where $t_{0}$ is the present age of the universe. In addition, the integral in Eq. (7) (see also Eq10) now takes the form $G(\alpha)=-\kappa Z(t)$ and $Z(t)=\int_{t_{0}}^{t} \alpha^{-3} e^{-2 \kappa C_{1}\left(t-t_{0}\right)}$. We note that at the present time we have $G(1)=0$. Obviously, using the above formula, the global density evolution (Eq.7) can be written

$$
\rho(\alpha)=C_{1}+\alpha^{-3} \frac{e^{-2 \kappa C_{1}\left(t-t_{0}\right)}}{\left[C_{2}-G(\alpha)\right]} .
$$

As expected, at early enough times $(t \rightarrow 0)$ the overall density scales according to $\rho(\alpha) \propto a^{-3}$, while close to the present epoch the density evolves according to

$$
\rho(\alpha) \simeq C_{1}+\frac{\alpha^{-3}}{C_{2}}
$$

which is approximately equivalent to the corresponding evolution in the $\Lambda$ cosmology in which the term $C_{1}$ resembles the constant-vacuum term $\left(\rho_{\Lambda}\right)$ and the $1 / C_{2}$ term resembles the density of matter $\left(\rho_{m}\right)$. We note that the effective pressure term (Eq. 3), for $\kappa \rightarrow 0$, becomes $\Psi \sim 3 C_{1} H$, which implies that: $P \sim-\Psi / 3 H=-C_{1}$. Therefore, this case relates to the traditional $\Lambda$ cosmology, since $C_{1}$ corresponds to $\rho_{\Lambda}$ (see Eq. 16). We now investigate in detail the dynamics of the $n=0$ model.

From Eq.(15), using the usual unit-less $\Omega$-like parametrization, we derive after some algebra that

$$
\left(\frac{H}{H_{0}}\right)^{2}=\Omega_{1,0}+\frac{\Omega_{1,0} \Omega_{2,0} \alpha^{-3} e^{-2 \kappa C_{1}\left(t-t_{0}\right)}}{\Omega_{1,0}+\kappa C_{1} \Omega_{2,0} Z(t)},
$$




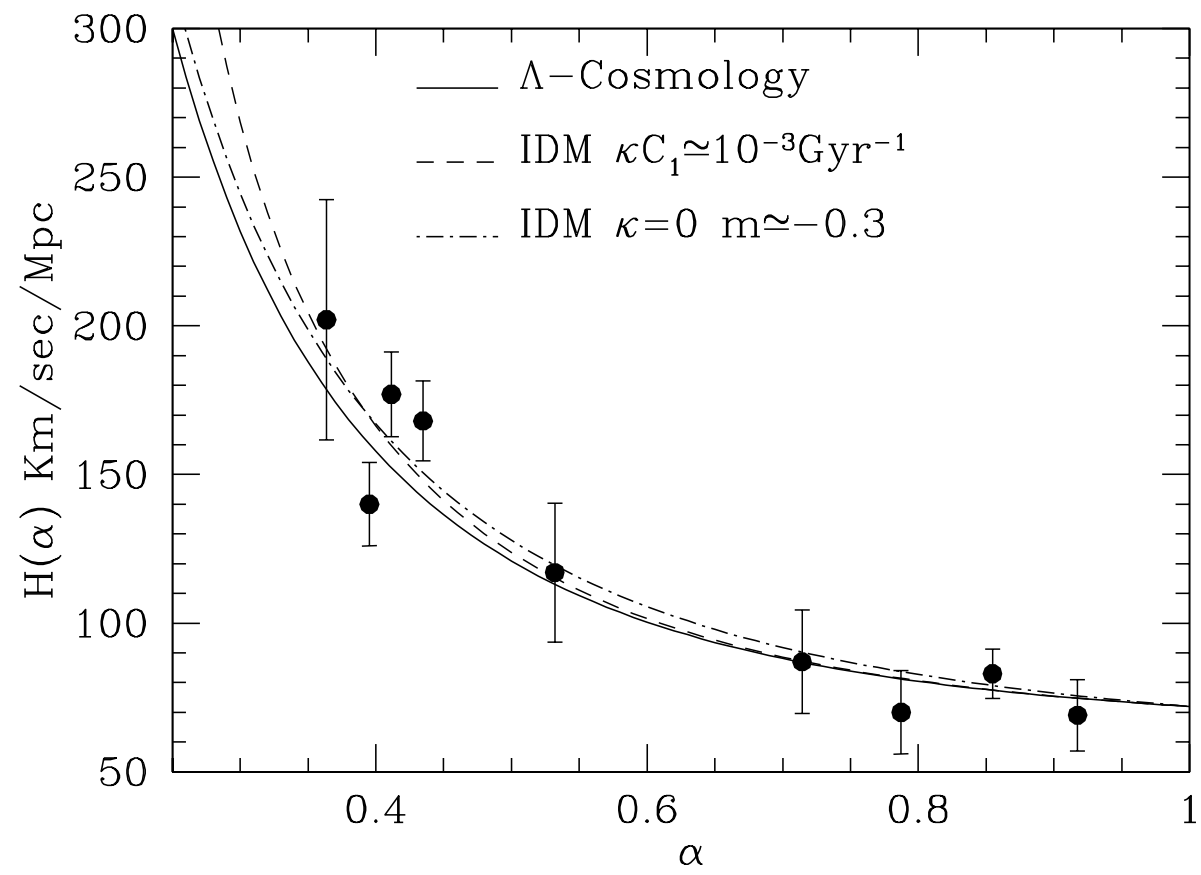

FIGURE 2. Comparison of the Hubble function provided by the traditional $\Lambda$ CDM model, which coincides with our $m=0$ model (for the best-fit model of the two free parameters - see text). The dashed line corresponds to our $m=0$ IDM model for the highest $\kappa C_{1}$ bound, provided by our fitting procedure $\left(\sim 10^{-3}\right)$. The dot-dashed line corresponds to our $\kappa=0$ IDM model for the best-fit model parameters $\left(m \simeq-0.3\right.$ and $\Omega_{2,0} \simeq 0.28$ ). Finally, the points correspond to the observational data ([28]).

where $\Omega_{1,0}=8 \pi G C_{1} / 3 H_{0}^{2}$ and $\Omega_{2,0}=8 \pi G / 3 H_{0}^{2} C_{2}$, which in the usual $\Lambda$ cosmology relates to $\Omega_{\Lambda}$ and $\Omega_{\mathrm{m}}$, respectively.

We can now attempt to compare the Hubble function of Eq.(17) to that corresponding to the usual $\Lambda$ model. To this end, we use a $\chi^{2}$ minimization between the different models (our IDM Eq 17 or the traditional $\Lambda$ CDM model) and the observed Hubble relation derived directly from early-type galaxies at high redshifts ([28]). For the case of our IDM model, we simultaneously fit the two free parameters of the model, i.e., $\Omega_{2,0}$ and $\kappa C_{1}$ for a flat background $\left(\Omega_{1,0}=1-\Omega_{2,0}\right)$ with $H_{0}=72 \mathrm{~km} / \mathrm{sec} / \mathrm{Mpc}$ and $t_{0}=H_{0}^{-1} \simeq 13.6$ Gyrs which is roughly the age of the universe of the corresponding $\Lambda$ cosmology. This procedure yields the best-fit model parameters $\Omega_{2,0}=0.3_{-0.08}^{+0.05}$ and $\log \left(\kappa C_{1}\right) \simeq-9.3$ (with a stringent upper limit $\simeq-3$, but unconstrained towards lower values) where $\chi^{2} /$ d.f. $=1.29$ (see left panel of Fig. 1). Using Eq.(4) we can now relate the range of values of $\kappa C_{1}$ to the mass of the DM particle, from which we obtain that

$$
M_{x}=\frac{1.205 \times 10^{-12}}{\kappa C_{1}} \frac{\langle\sigma u\rangle}{10^{-22}} \mathrm{GeV},
$$

(see also right panel of Fig. 1) and since $\kappa C_{1}$ is unbound at small values, it is consistent with currently accepted lower bounds of $M_{x}(\sim 10 \mathrm{GeV})$ (see [29] and references therein). The corresponding Hubble relation (Fig. 2), provided by the best-fit model free parameters, is indistinguishable from that of the traditional $\Lambda$ CDM model, because of the very small value of $\kappa C_{1} \simeq 10^{-9.3}$. For completeness, we also show, as the dashed line, the IDM solution provided by $M_{x} \sim 1 \mathrm{eV}\left(\kappa C_{1} \simeq 10^{-3}\right)$, which is the stringent lower bound found by our analysis. In this case, the predicted Hubble expansion deviates significantly from the traditional $\Lambda$ model at small $\alpha$ values indicating that it would probably create significant alterations to the standard BBN (see [30] and references therein).

Although the present analysis does not provide any important constraints on $M_{x}$ (within our model), we plan on the future to use a large amount of cosmologically relevant data to attempt to place stronger $M_{x}$ constraints. 


\section{MIMICKING THE QUINTESSENCE DARK ENERGY}

In this case, we assume that the annihilation term is negligible $[\kappa=0$ and $f(\alpha)=0$ and $F(\alpha)=1]$ and the particle creation term dominates. This situation is mathematically equivalent to the gravitational DM particle creation process within the context of non-equilibrium thermodynamics [26], the important cosmological consequences of which were studied by [21] (see also references therein).

Using our nomenclature it is straightforward to obtain the evolution of the total energy density from Eq.(7), as:

$$
\rho(\alpha)=D \alpha^{-3}+C_{1} \alpha^{m},
$$

where $D=1 / C_{2}$. The conditions in which the current model acts as a quintessence cosmology, are given by $C_{1}>0$, $C_{2}>0$, and $w_{\mathrm{IDM}}=-1-m / 3$, which implies that to have an inflection point, the following should be satisfied: $w_{\text {IDM }}<-1 / 3$ or $m>-2$ (see section 2.1). We note, that the Hubble flow is now given by

$$
\left(\frac{H}{H_{0}}\right)^{2}=\Omega_{2,0} \alpha^{-3}+\Omega_{1,0} \alpha^{m}
$$

where $\Omega_{2,0}=8 \pi G D / 3 H_{0}^{2}$ and $\Omega_{1,0}=8 \pi G C_{1} / 3 H_{0}^{2}$. Finally, by minimizing the corresponding $\chi^{2}$, we find that the best-fit model values are $\Omega_{2,0} \simeq 0.28$ and $m \simeq-0.30\left(w_{\text {IDM }} \simeq-0.90\right)$ with $\chi^{2} /$ d.f. $=1.29$. The corresponding Hubble flow curve is shown in Fig. 2 as the dot-dashed line. We note that this solution is mathematically equivalent to that of the gravitational matter creation model of [21].

\section{CONCLUSIONS}

In this work we investigate analytically the evolution of the global density of the universe in the context of an interacting DM scenario by solving analytically the collisional Boltzmann equation in an expanding Universe. The possible disequilibrium between the DM particle creation and annihilation processes, regardless of its cause and in which the particle creation term dominates, creates an effective source term with negative pressure which (acting as dark energy) provides an accelerated expansion phase of the scale factor. Finally, comparing the observed Hubble function of a few high-redshift elliptical galaxies with that predicted by our simplest IDM model $(m=0)$, we find that the effective annihilation term is quite small.

\section{APPENDIX A: THE MAIN ASSUMPTIONS OF OUR MODEL}

The reason why a cosmological constant or a component mimicking it leads to a cosmic acceleration is because it introduces in Friedmann's equation, which governs the global dynamics, a component which has an equation of state with negative pressure. Our model creates exactly the equivalent to the above, ie., an effective source term which has negative pressure. The avenue throw which this is accomplished is via the collisional Boltzmann equation in an expanding universe (eg. [25]) in which the disequilibrium between the annihilation $\left(\kappa \rho^{2}\right)$ and particle creation $(\Psi)$ processes provides the effective pressure: $P=\left(\kappa \rho^{2}-\Psi\right) / 3 H$, which when negative acts exactly as a repulsive force, and therefore provides a cosmic acceleration. The basic assumption of this work is that the DM fluid is non-perfect, ie., there is a disequilibrium between the particle pair annihilation and creation processes. Note that in our general solution (eq7) we do not specify the direction of this disequilibrium. Only in the detailed analysis we seek solutions which provide an accelerated phase of the universe (for which of course one condition is $P<0$ ), which we indeed find.

\section{APPENDIX B: SOLUTIONS OF THE RICCATI EQUATION}

With the aid of the differential equation theory we present solutions that are relevant to our eq.(4). In general a Riccati differential equation is given by

$$
y^{\prime}=f(x) y^{2}+g(x) y+R(x)
$$

and it is fully solvable only when a particular solution is known. Below we present two case in which analytical solutions are possible.

- Case 1: For the case where: 


$$
R(x)=C_{1} n x^{n-1}-C_{1}^{2} x^{2 n} f(x)-C_{1} x^{n} g(x)
$$

the particular solution is $x^{n}$ and thus the corresponding general solution can be written as:

$$
y(x)=C_{1} x^{n}+\Phi(x)\left[C_{2}-\int_{1}^{x} f(u) \Phi(u) d u\right]^{-1}
$$

where

$$
\Phi(x)=\exp \left[\int_{1}^{x}\left(2 C_{1} u^{n} f(u)+g(u)\right) d u\right]
$$

and $C_{1}, C_{2}$ are the integration constants. Using now eq. (5) we get $\Psi(x)=x H(x) R(x)=C_{1}(n+3) x^{n} H(x)+\kappa C_{1}^{2} x^{2 n}$.

- Case 2: For the case where:

$$
R(x)=h^{\prime}(x) \text { with } g(x)=-f(x) h(x)
$$

the particular solution is $h(x)$ [in our case we have $h(x)=-3 \kappa^{-1} H(x)$ ]. The general solution now becomes:

$$
y(x)=h(x)+\Phi(x)\left[C_{2}-\int_{1}^{x} f(u) \Phi(u) d u\right]^{-1}
$$

where

$$
\Phi(x)=\exp \left[\int_{1}^{x} f(u) h(u) d u\right] .
$$

In this framework, using eq. (5) we finally get $\Psi(x)=x H(x) R(x)=-3 \kappa^{-1} x H(x) H^{\prime}(x)$.

Note that the solution of Case 1 is the only one providing a $\propto \alpha^{-3}$ dependence of the scale factor (see eqs. 15 , 16 and 19.

\section{APPENDIX C: JUSTIFICATION OF THE FUNCTIONAL FORM OF $\Psi$}

We assume that we have a non-perfect cosmic fluid in a disequilibrium phase with energy density $\rho$ then from the collisional Boltzmann equation, we have that

$$
\Psi=\dot{\rho}+3 H \rho+\kappa \rho^{2}=\frac{\mathrm{d} \rho}{\mathrm{d} a} a H+3 H \rho+\kappa \rho^{2} .
$$

Furthermore, we assume that for a convenient period of time, the cosmic fluid, in an expanding Universe, is slowly diluted according to $\rho \sim C_{1} \alpha^{m}$ ( $m \leq 0$ ). From a mathematical point of view, the latter assumption simply means that a solution of the form $\propto \alpha^{m}$ is a particular solution of the Boltzmann equation. Therefore, we have finally that:

$$
\Psi \simeq C_{1}(m+3) a^{m} H+\kappa C_{1}^{2} a^{2 m} .
$$

\section{REFERENCES}

1. D.N. Spergel, et al., Astrophys. J. Suplem., 170, 377, (2007)

2. T.M. Davis et al., Astrophys. J., 666, 716, (2007)

3. M. Kowalski, et al., Astrophys. J., 686, 749, (2008)

4. E. Komatsu, et al., Astrophys. J. Suplem., 180, 330, (2009)

5. S. Weinberg, in The cosmological constant problem, Reviews of modern physics, 61, 1, (1989)

6. C. Wetterich, Astron. Astrophys. 301, 321 (1995)

7. R. R. Caldwell, R. Dave, and P. J. Steinhardt P. J., Phys. Rev. Lett., 80, pp 1582, (1998)

8. P. J. Peebles and B. Ratra, in The cosmological constant and dark energy, Reviews of modern physics , 75, p. 559-606, (2003)

9. P. Brax and J. Martin, Phys. Lett. B468, 40 (1999)

10. A. W. Brookfield, C. van de Bruck, D. F. Mota, and D. Tocchini-Valentini, Phys. Rev. Lett. 96, 061301 (2006)

11. C. G. Boehmer and T. Harko, Eur. Phys. J. C50, 423 (2007) 
12. L. Amendola and D. Tocchini-Valentini, Phys. Rev. D 64, 043509 (2001); W. Zimdahl, D. Pavón, L. P. Chimento, Phys.Lett.B, 521, 133, (2001)

L. Amendola, C. Quercellini, D. Tocchini-Valentini and A. Pasqui, Astrophys. J. 583, L53 (2003); G. Mangano, G. Miele and V. Pettorino, Mod. Phys. Lett. A 18, 831 (2003); L. P. Chimento, A. S. Jakubi, D. Pavón, W. Zimdahl, Phys.Rev.D, 67, 083513, (2003)

13. R. G. Cai and A. Wang, JCAP 0503, 002 (2005); J. B. Binder and G. M. Kremer, Gen. Rel. Grav. 38, 857 (2006); G. Huey and B. D. Wandelt, Phys. Rev. D 74, 023519 (2006); B. Wang, C-Y, Lin, Abdalla, Phys. Lett. B., 637, 357, (2006); R. Mainini, S. Bonometto, Phys.Rev.D, 74, 043504, (2006); S. Das, P. S. Corasaniti, J. Khoury, Phys. Rev. D. 73, 083509, (2006); G. M. Kremer, Gen.Relativ.Gravit., 39, 965, (2007); A. W. Brookfield, C. van de Bruck, and L. M. H., Hall,[arXiv:0709.2297], (2007); J.-H He, B. Wang, B., JCAP, 06, 010, (2008)

14. D.N. Spergel and P.J. Steinhardt, Phys.Rev.Lett. 84, 3760 (2000)

15. R.A. Flores, J.R. Primack, Astrophys. Journal, 427, L1 (1994)

16. B. Moore et al. Astrophys. Journal, 524, L19 (1999)

17. D.. Hooper, D. P., Finkbeiner, G. Dobler, Phys. Rev. D., 76, 3012, (2007)

18. M. Regis, P. Ullio, Phys. Rev. D., 78, 3505, (2008)

19. W. Zimdahl, D.J. Schwarz, A.B. Balakin, D. Pavon, Phys. Rev. D 64, 3501 (2001);

20. A.B. Balakin, D. Pavon, D.J. Schwarz, W. Zimdahl, N.J.Phys, 5, 85 (2003).

21. J. A. S. Lima, F. E. Silva, R. C. Santos, Class. and Quantum Gravity, 25, 205006, (2008)

22. G. R. Farrar and P. J. E. Peebles, Astrophys. J. 604, 1 (2004)

23. S. S. Gubser and P. J. E. Peebles, Phys. Rev. D70, 123510 (2004)

24. S. Basilakos, \&, M. Plionis, Astron. \& Astrophysics, in press, arXiv: 0807.4590 (2009)

25. E. W, Kolb \& M. S. Turner, The Early Universe, Addison-Wesley Publishing, (1990)

26. I. Prigogine, J. Geheniau, E. Gunzig, P. Nardone, Gen. Rel.Grav. 21, 767, (1989); M. O. Calvao, J. A. S. Lima, I. Waga, Phys.Lett. A, 162, 223, (1992); J. A. S., Lima, A. S. M. Germano, L. R. W. Abramo, Phys.Rev. D., 53, 4287, (1996)

27. S. Weinberg, in Cosmology, Oxford University Press, 2008

28. J., Simon, L. Verde, R. Jimenez, Phys. Rev. D., 71, 123001, (2005)

29. M. Cirelli, F. Iocco, P. Panci, JCAP, 10, 9, (2009)

30. F. Iocco, et al. Phys.Rept., 472, 1, (2009) 